\title{
Simulation of Wind Power Integration with Modular Multilevel Converter-Based High Voltage Direct Current
}

\author{
Hua LI, Keqilao MENG*, Xiaoyan LI, Yufei PENG
}

\begin{abstract}
The growing demand for large-capacity long distance transmission of wind power has boosted the development of flexible direct current (DC) transmission technology. To facilitate wind power integration, this paper designs a modular multilevel converter (MMC) for steady-state operation, using the parameters of the demonstration DC transmission project of offshore wind power in Sheyang County, eastern China's Jiangsu Province. Relying on the simulation platform of PSCAD/EMTDC, the authors analyzed the proposed control theory, and verified that, under different working conditions (e.g., changing wind speed), the MMC-based high voltage direct current (MMC-HVDC) transmission system can integrate the wind power safely and efficiently. In addition, the authors discussed how to enhance the fault ride-through (FRT), a prominent problem in wind power operation, of the flexible DC system containing wind power, from the perspective of alternating current (AC) fault and DC fault.
\end{abstract}

Keywords: fault ride-through (FRT); modular multilevel converter (MMC); wind power integration

\section{INTRODUCTION}

In 2021, the Chinese government released its latest work report on the back-to-back meetings of the country's top two political bodies, making the pledge to set a peak for carbon emissions and pursue carbon neutrality. The main paths for achieving net-zero carbon emissions include preparing an action plan for peaking carbon emissions before 2030, optimizing industrial and energy structures, promoting clean and efficient use of coal, and developing power generation by new energies (e.g., wind power)[1]. China plans to increase its total installed capacity of wind power to 540 million $\mathrm{kW}$ by 2025 .

The 14th Five-Year Plan issued by the Global Energy Internet Development Cooperation Organization in 2020 points out that the installed capacity of wind power is 1.967 billion kilowatts, and the power generation is 4.36 trillion kilowatts, accounting for $30.5 \%$ of the total power generation, making it the largest energy source in my country [1]. Wind energy resources in China concentrate in the northern part, which is far away from the major power consumers on the southeastern coast. The mismatch between the distributions of wind energy resources and power demand hinders the integration and consumption of wind power, resulting in severe wind curtailment. It also means that a large volume of wind power needs to be transmitted over a long distance. According to the St. Clair curve, the alternating current (AC) is not suitable for long distance transmission. Therefore, high voltage direct current (HVDC) becomes the preferred technology [2].

Modular Multilevel Converter (MMC) has received extensive attention and research due to its modular design, lower voltage harmonic content and smaller converter station losses. The MMC-HVDC system has good wind power grid-connection capability and strong antiinterference ability. The application of MMC-HVDC technology to wind farm interconnection can effectively improve the stable operation characteristics of wind farms [3].

This paper attempts to solve the key problem of large scale wind power integration via MMC-HVDC system. Firstly, the design of a single doubly-fed induction generator (DFIG) controller was briefly introduced, and an equivalent wind farm was established with controlled current source. Next, the MMC was mathematically modeled, and the double closed-loop control, which is based on DC control, was adopted for the grid-side MMC (GSMMC), according to the different objectives of converter control: the outer ring is the power ring, and the inner ring is the current ring. Meanwhile, the AC voltage and frequency control was selected for the wind farm-side MMC (WFMMC). The proposed control strategy was proved correct on the simulation platform of PSCAD/EMTDC. Finally, the authors discussed the $\mathrm{AC} / \mathrm{DC}$ fault ride-through (FRT), a prominent issue in largescale wind power integration via flexible DC system.

\section{WIND POWER INTEGRATION VIA FLEXIBLE DC SYSTEM}

With reference to the offshore wind power DC transmission demonstration project in Sheyang County, Jiangsu, a simulation model of the wind farm as shown in Fig. 1 through the MMC-HVDC gridconnected system was established to verify the correctness of the established model.

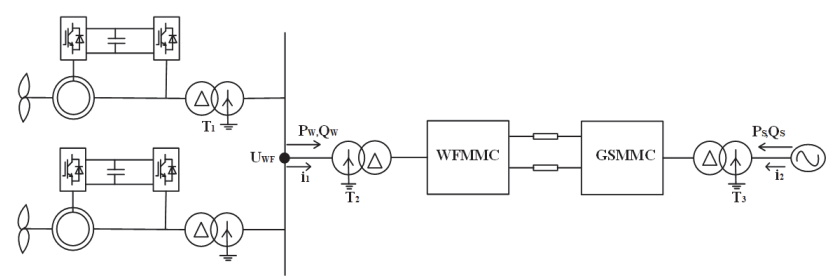

Figure 1 Structure of MMC-HVDC-based wind power integration system

Note: $P_{\mathrm{W}}$ and $Q_{\mathrm{w}}$ are the active and passive powers transmitted from the wind farm to the DC system, respectively; $P_{\mathrm{S}}$ and $Q_{\mathrm{S}}$ are the active and passive powers exchanged between the wind farm and the DC system, respectively; $i_{1}$ and $i_{2}$ are the AC currents of WFMMC and GSMMC, respectively; $U_{\mathrm{WF}}$ is the outlet voltage vector of the wind farm; $T_{2}$ and $T_{3}$ are the converter transformers of WFMMC and GSMMC, respectively. The two converter transformers adopt the Star-Delta $(Y d)$ connection to isolate the zero-sequence circuit of the $\mathrm{AC}$ system. 


\section{DESIGN OF DFIG CONTROLLER}

The design of dual-fed induction wind turbine controllers has been detailed by Chen [4]. The rotor-side converter uses the stator flux-oriented vector control, while the grid-side converter adopts the grid voltage-oriented vector control. The active and reactive powers imported by DFIG stator winding to the $\mathrm{AC}$ system are regulated through decoupling control. Considering the needs of this research, the wake effect of wind power generator and the differences induced by factors like wind direction were both ignored. To simplify the wind farm model and speed up simulation, some generators were replaced with controlled current sources to simulate a large wind farm.

\section{DESIGN AND CONTROL OF MMC-HVDC MODEL FOR WIND POWER INTEGRATION}

As shown in Fig. 2, the MMC has six bridge arms in three phases. Each phase unit encompasses an upper bridge arm, and a lower bridge arm. Each bridge arm consists of $\mathrm{n}$ sub-modules (SMs) connected in series with an electric reactor $L_{\mathrm{S}}$. In every SM, there is a half-bridge composed of two insulated gate bipolar transistors (IGBTs) with two antiparallel diodes, and an energy storage capacitor. The cut-in, cut-out, and latched states of the SMs can be switched by controlling the on and off states of $V T_{1}$ and $V T_{2}$. According to Eq. (1), it can be seen that the output AC voltage can be adjusted by changing the number of cut-in SMs on the upper and lower bridge arms. To keep the stability of AC voltage, the number of cut-in SMs in each phase unit must be maintained at $N$. Therefore, the MMC outputs a total of $N+1$ levels.

$$
N=\frac{U_{\mathrm{dc}}}{U_{\mathrm{c}}}
$$

$U_{\mathrm{dc}}$ is the voltage on the DC side of the MMC, $U_{\mathrm{c}}$ is the voltage of each sub-module, $N$ is the number of submodules.

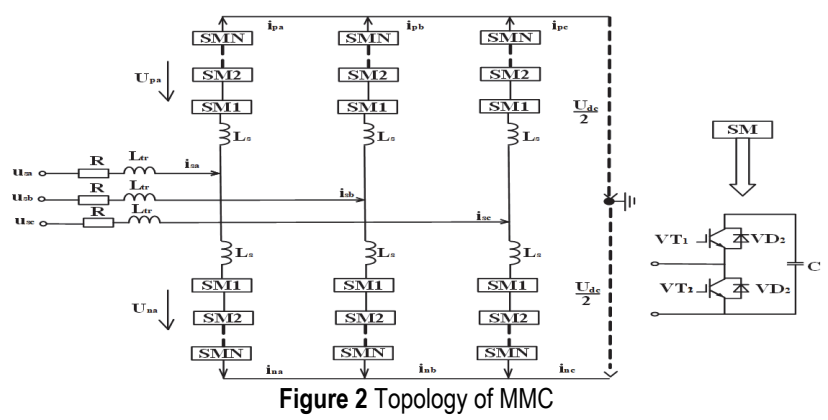

Note: $u_{\mathrm{sa}}, u_{\mathrm{sb}}$ and $u_{\mathrm{sc}}$ are the fundamental voltage components of the three-phase buses in the AC system, respectively; $i_{\mathrm{sa}}, i_{\mathrm{sb}}$ and $i_{\mathrm{sc}}$ are the fundamental current components of the three-phase buses in the AC system, respectively; $L$ is the sum of the parallel inductance of the upper and lower bridge arms and the stray inductance of converter transformer; $R$ is the equivalent resistance of converter transformer and bridge arm resistance; $u_{\mathrm{ca}}, u_{\mathrm{cb}}$ and $u_{\mathrm{cc}}$ are the fundamental components of the three-phase voltages outputted by the converter.
The equivalent circuit of the MMC can be simplified as in Fig. 3.

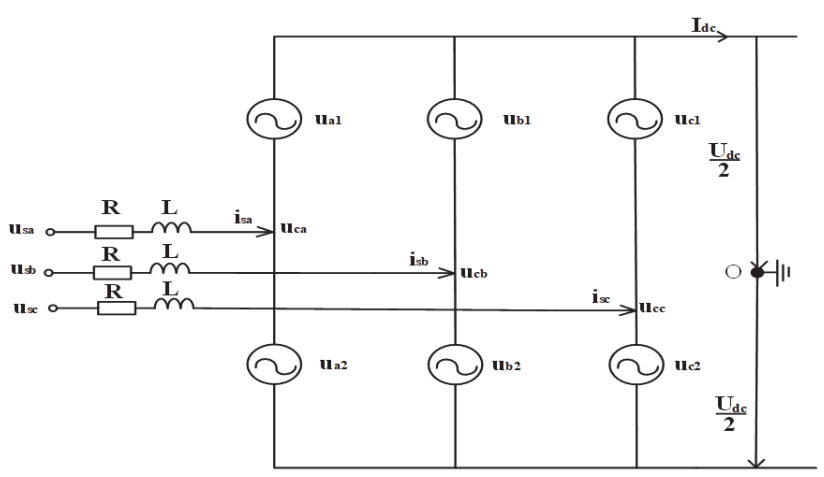

Figure 3 Simplified equivalent circuit of MMC

From Fig. 3, the mathematical model of the MMC in the three-phase static coordinate system can be derived as:

$\left\{\begin{array}{l}L \frac{\mathrm{d} i_{\mathrm{sa}}}{\mathrm{d} t}+i_{\mathrm{sa}} R=u_{\mathrm{sa}}-u_{\mathrm{ca}} \\ L \frac{\mathrm{d} i_{\mathrm{sb}}}{\mathrm{d} t}+i_{\mathrm{sb}} R=u_{\mathrm{sb}}-u_{\mathrm{cb}} \\ L \frac{\mathrm{d} i_{\mathrm{sc}}}{\mathrm{d} t}+i_{\mathrm{sc}} R=u_{\mathrm{sc}}-u_{\mathrm{cc}}\end{array}\right.$

\subsection{Design of GSMMC Controller}

During steady-state operation, the GSMMC control aims to keep the DC line voltage stable, and provide necessary reactive support to the AC system. The GSMMC adopts the DC control strategy, which encompasses a power controller in the outer loop and a current controller in the inner loop. Suppose the $d$-axis of the synchronous rotation coordinate system is of the same direction of the rotation vector of grid voltage. Then, the AC-side transient state of GSMMC in the $d-q$ coordinate system can be modeled by $[5,6]$ :

$$
\left\{\begin{array}{l}
L \frac{\mathrm{d} i_{d}}{\mathrm{~d} t}=u_{\mathrm{s} d}-R_{i d}-u_{\mathrm{c} d}-w L_{i q} \\
L \frac{\mathrm{d} i_{q}}{\mathrm{~d} t}=u_{\mathrm{s} q}-R_{i q}-u_{\mathrm{c} q}+w L_{i d}
\end{array}\right.
$$

where, $u_{\mathrm{s} d}$ and $u_{\mathrm{s} q}$ are the $d$-axis and $q$-axis components of the bus voltage in the AC system of the grid, respectively; $u_{\mathrm{c} d}$ and $u_{\mathrm{c} q}$ are the $d$-axis and $q$-axis components of the converter valve-side voltage of GSMMC, respectively; $i_{d}$ and $i_{q}$ are the $d$-axis and $q$-axis components of the current flowing into the converter valve of GSMMC, respectively; $\omega$ is the angular frequency of the synchronous rotation coordinate system, that is, the angular frequency of the bus voltage in the $\mathrm{AC}$ system of the grid.

It can be seen that the current of the $\mathrm{AC}$ system is affected by the control quantities $u_{\mathrm{c} d}$ and $u_{\mathrm{c} q}$, grid voltages $u_{\mathrm{s} d}$ and $u_{\mathrm{s} q}$, and coupling items $\omega L_{i q}$ and $\omega L_{i d}$. Therefore, it is impossible to independently control the variables on the $d$-axis and $q$-axis. To solve the problem, the feed forward decoupling strategy needs to be introduced to eliminate the coupling items of $d$-axis and $q$-axis currents: 
$\left\{\begin{array}{l}u_{\mathrm{c} d}=u_{d}^{*}-w L_{i q}+u_{\mathrm{s} d} \\ u_{\mathrm{c} q}=u_{q}^{*}+w L_{i d}+u_{\mathrm{s} q}\end{array}\right.$

$\left\{\begin{array}{l}u_{\mathrm{d}}^{*}=L \frac{\mathrm{d} i_{\mathrm{d}}}{\mathrm{d} t}+R i_{\mathrm{d}}=-\left(K_{\mathrm{p} 1}+\frac{K_{\mathrm{i} 1}}{s}\right)\left(i_{\mathrm{dref}}-i_{\mathrm{d}}\right) \\ u_{\mathrm{q}}^{*}=L \frac{\mathrm{d} i_{\mathrm{q}}}{\mathrm{d} t}+R i_{\mathrm{q}}=-\left(K_{\mathrm{p} 2}+\frac{K_{\mathrm{i} 2}}{s}\right)\left(i_{\mathrm{qref}}-i_{\mathrm{q}}\right)\end{array}\right.$

where, $K_{\mathrm{p} 1}, K_{\mathrm{p} 2}$ and $K_{\mathrm{i} 1}, K_{\mathrm{i} 2}$ are the proportional and integral coefficients of the inner loop current controller, respectively; $i_{\text {dref }}$ and $i_{\text {qref }}$ are the reference active and reactive currents of the inner ring, respectively. The reference values are given by the outer loop controller. According to the control objective of the GSMMC, the outer loop controller adopts the reactive power control with fixed DC current:

$$
\left\{\begin{array}{c}
i_{\mathrm{dref}}=\left(K_{\mathrm{p} 3}+\frac{K_{\mathrm{i} 3}}{s}\right)\left(U_{\mathrm{dcref}}-U_{\mathrm{dc}}\right) \\
i_{\mathrm{qref}}=\left(K_{\mathrm{p} 4}+\frac{K_{\mathrm{i} 4}}{s}\right)\left(Q_{\mathrm{ref}}-Q\right)
\end{array}\right.
$$

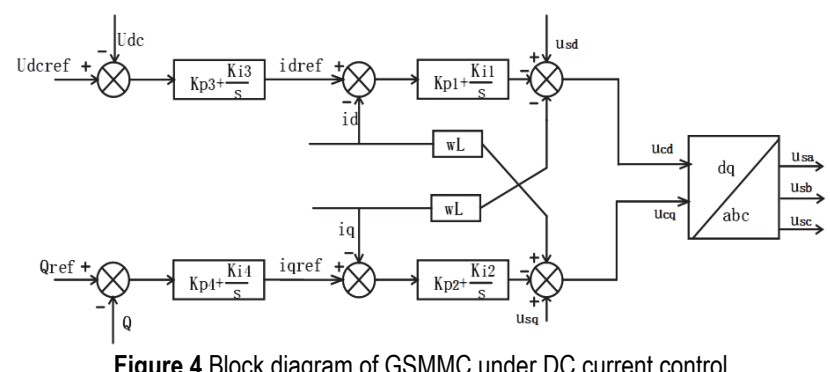

Fig. 4 shows the block diagram of GSMMC under DC current control.

\subsection{Design of WFMMC Controller}

WFMMC control aims to transmit all the power generated by the wind farm to high voltage DC transmission lines. Without a strong enough rotary power supply for frequency support, the wind farm can be generally treated as a passive network. The AC grid of the wind farm needs to be supported by the WFMMC, which stabilizes the AC voltage amplitude and frequency (i.e., implements $V / f$ control) of the current collection system at the wind farm. Despite having the same control objective, the $V / f$ controllers might have different structures [7]. This paper selects the controller shown in Fig. 5.

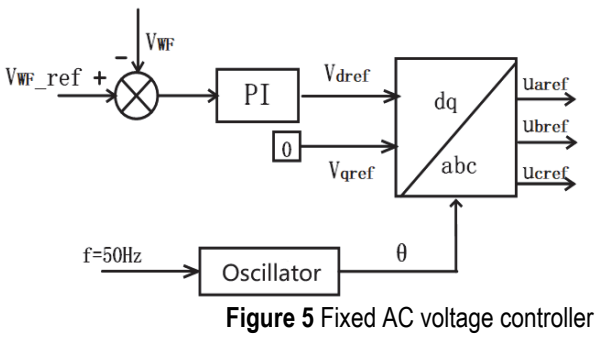

The reference AC voltage outputted by the wind farm $V_{\text {WF_ref }}$ was subtracted from the effective measured voltage $V_{\mathrm{WF}}$. The difference was passed through the proportionalintegral (PI) controller to generate the reference wave of daxis voltage. Taking $V_{\text {qref }}=0.50 \mathrm{~Hz}$ frequency was passed through an oscillator to produce the initial phase angle $\theta$ of the fundamental voltage outputted by the WFMMC. Finally, the $2 r / 3 s$ transform was implemented to obtain the AC-side reference voltage waveform [8].

\section{SIMULATION OF WIND POWER INTEGRATION VIA FLEXIBLE DC SYSTEM}

In the simulation environment of PSCAD/EMTDC, a simulation model was constructed for wind power generation based on MMC-HVDC. A total of 200 DFIGs was deployed. The generator parameters were configured as follows: rated power $5 \mathrm{MW}$, rated voltage $0.69 \mathrm{kV}$, rated frequency is $50 \mathrm{~Hz}$; stator-side resistance $0.0054 \mathrm{Pu}$, rotorside resistance $0.0061 \mathrm{Pu}$; stator-side self-inductance 0.10 $\mathrm{Pu}$, rotor-side self-inductance $0.11 \mathrm{Pu}$.

The MMC-HVDC system parameters were configured as follows: rated capacity of the converter station 1000 mVA, DC voltage level $\pm 250 \mathrm{kV}, \mathrm{SM}$ number of upper and lower bridge arms 100 , SM capacitance is $6800 \mathrm{uF}$, inductive reactance of bridge arm $30 \mathrm{mH}$, and SM capacitor voltage $5 \mathrm{kV}$.

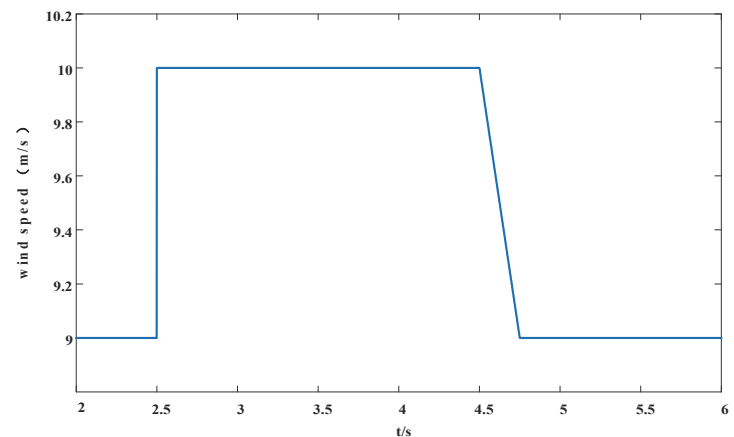

(a)Wind speed

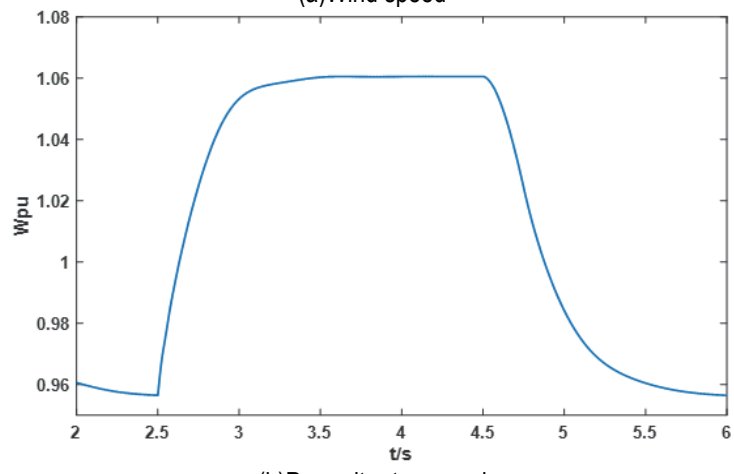

(b)Per-unit rotor speed

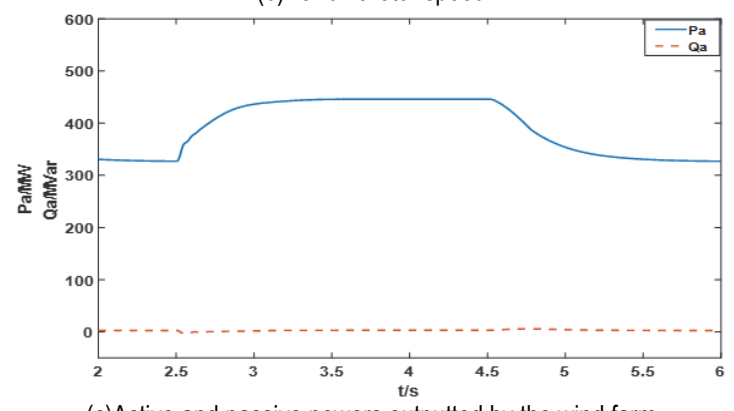

(c)Active and passive powers outputted by the wind farm Figure 6 Simulation results at changing wind speed 
The MMC adopts the carrier phase shifted-sinusoidal pulse width modulation (CPS-SPWM), and capacitance voltage equalization control [9]. The WFMMC adopts the fixed $\mathrm{AC}$ voltage and fixed frequency control strategy. The rated voltage at the point of common contact (PCC) of the sending end was set to $220 \mathrm{kV}$; the frequency of the $\mathrm{AC}$ system was set to $50 \mathrm{~Hz}$. The GSMMC adopts the fixed DC voltage and fixed reactive power control strategy. The DC voltage was set to $500 \mathrm{kV}$; the reactive power was set to 0 Mvar. The reference positive directions of active and reactive powers are shown in Fig. 1.

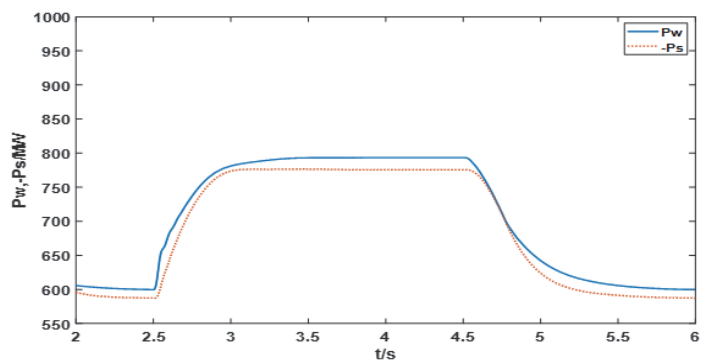

(a) Active power $P w$ outputted by the wind farm and the active power $P s$ transmitted to the $\mathrm{AC}$ system

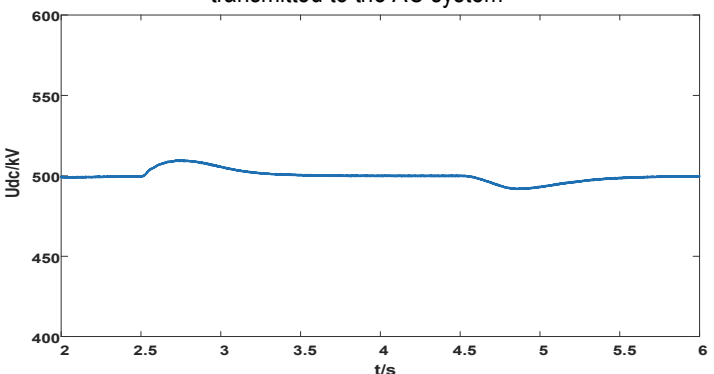

(b) DC voltage $U_{\mathrm{dc}}$

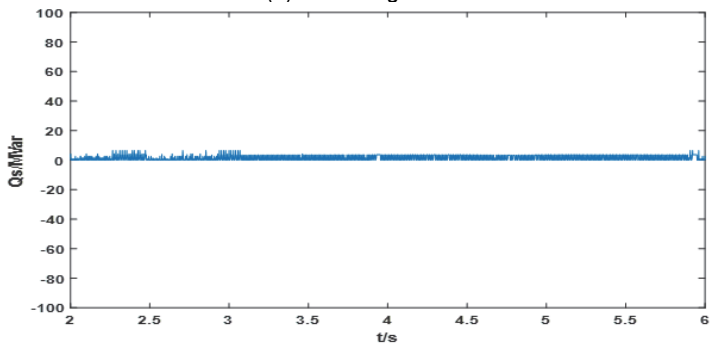

(c) Reactive power Qs absorbed by the MMC

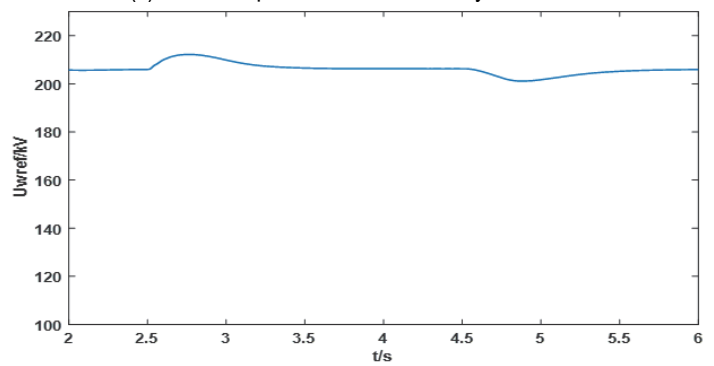

(d) Effective AC voltage at the PCC of the wind farm

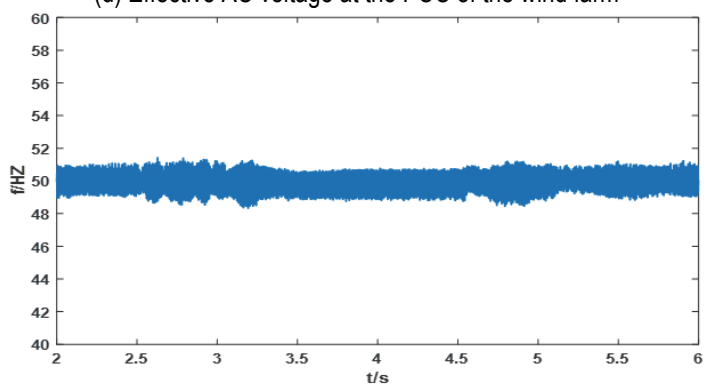

(e) AC frequency of current collection system at the wind farm Figure 7 Simulation results of WFMMC and GSMMC
The following simulation was carried out to verify the correctness of the MMC-based wind power integration simulation model. The wind speed was increased abruptly from $9 \mathrm{~m} / \mathrm{s}$ to $10 \mathrm{~m} / \mathrm{s}$ at $2.5 \mathrm{~s}$, and gradually changed to 9 $\mathrm{m} / \mathrm{s}$ after $4.5 \mathrm{~s}$. Use this wind speed to study the stability of the grid-connected system. The speed never surpassed the rated wind speed. To harness as much wind energy as possible, the pitch angle was set to $0^{\circ}$, so that the rotor speed tracks the changing wind speed. The active and passive powers outputted by the generators were under decoupling control. As shown in Fig. 6, the active power changed with the variation in the captured wind energy, while the reactive power stabilized near the expected value of zero.

Fig. 7 shows the simulation results of WFMMC and GSMMC. Fig. 7a displays the active power $P_{\mathrm{W}}$ transmitted by WFMMC, and the active power $P_{\mathrm{s}}$ transmitted by GSMMC to the AC system. The $P$ s was slightly smaller than $P_{\mathrm{W}}$, due to the loss on the DC transmission lines. As shown in Figs. $7 \mathrm{~b}$ and $7 \mathrm{c}$, the DC voltage stabilized at around $500 \mathrm{kV}$, reactive power is controlled at $0 \mathrm{Mvar}$. The reactive power of GSMMC remained at zero, without being affected by fluctuations in active power. In this way, the converter station realizes decoupling control of active and reactive powers. As shown in Figs. 7d and 7e, the PCC of the wind farm oscillated slightly under the changing wind speed; the AC frequency of the current collection system at the wind far stabilized near the rated value. The above waveforms indicate that the MMC-HVDC can effectively transmit the active power outputted by the wind farm to the AC system, which verifies the correctness of the simulation model for the MMC-based wind power integration.

\section{FRT OF DC SYSTEM FOR WIND POWER INTEGRATION}

Besides setting up the system-level MMC-HVDCbased wind power integration control strategy, it is important to study the control measure against disturbance or fault, and ensure the FRT ability of the integration system. In fact, the FRT is a key metric of system stability, which has attracted much attention from scholars. The research of low voltage ride through (LVRT) of wind power generators is already mature. The FRT ability of the integration system is a research hotspot. The FRT of DC integration system can be divided into AC FRT and DC FRT.

\subsection{AC FRT}

AC FRT refers to the ability to maintain the connection between the converter station and the DC grid, and continue to transmit certain amount of power, when the $\mathrm{AC}$ grid is faulty. During wind power integration via MMCHVDC, if the voltage drops due to grid fault, fewer active power will be transmitted from the grid-side converter station to the $\mathrm{AC}$ grid. If no measure is taken, the wind farm will operate under the original control strategy, and feed all the power to the DC system. Then, the DC system will have excess power. The ensuing rise of DC voltage will threaten the safety of system operation.

To realize the FRT of wind power integration, the key lies in balancing the active power in the system during the 
fault. The existing methods fall into two categories: One category of methods adds a circuit to the DC side to consume the excess energy, e.g., the addition of unloading circuit; the other category of methods cuts down the output power of the wind farm, e.g., establishing fast communication, increasing frequency, and reducing voltage.

\subsubsection{Addition of Unloading Circuit}

The addition of unloading circuit essentially adds a controllable unloading resistor in parallel to the DC side. The resistor will enter operation once the DC voltage surpasses the preset threshold, and consumes the excess energy of the DC system in the form of thermal energy. Fig. 8 shows the most typical unloading circuit.

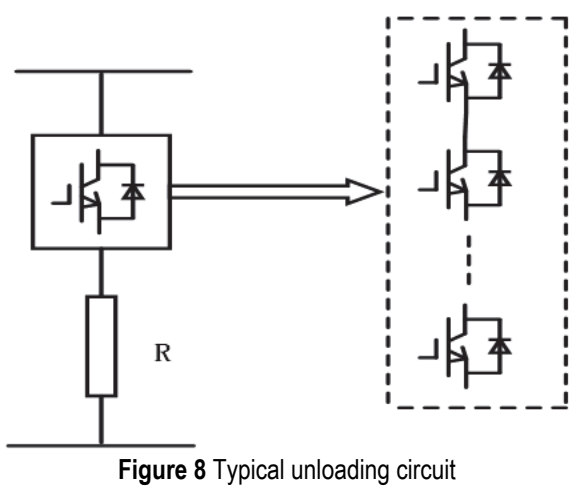

Liu et al. [10] explored the modular multilevel unloading circuit in Fig. 9a, analyzed the multilevel centralized unloading circuit in Fig. 9b, and proved that the two structures have similar FRT ability through simulation. The multilevel centralized unloading circuit inherits the merits of the modular multilevel unloading circuit, while avoiding the cost and heat dissipation problems of distributed resistors.

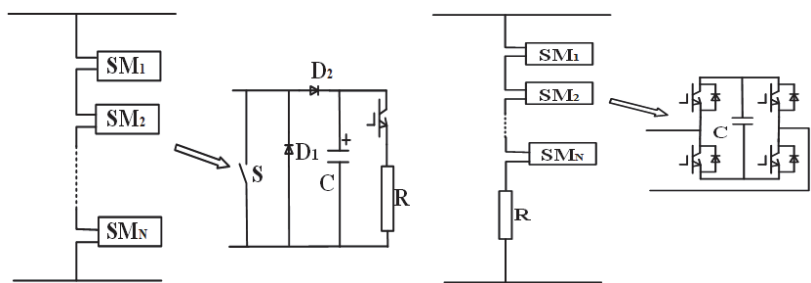

(a)Modular multilevel unloading circuit; (b) Multilevel centralized unloading circuit Figure 9 Topologies of different unloading circuits

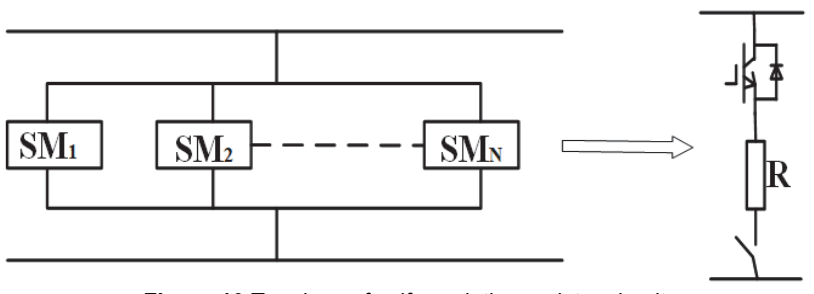

Figure 10 Topology of self-regulating resistor circuit

Drawing on the ideas of MMC SMs, Zhang et al. [10] proposed the self-regulating resistor circuit (Fig. 10). The number of SMs to be cut-in depends on the power difference. During the fault period, the fault of different degrees can be mitigated by cutting in different number of SMs.

\subsubsection{Establishing Fast Communication}

Based on the information transfer between the gridside converter station and the wind farm, the converter station will send a signal to the wind farm upon detecting a drop of the output active power; then, the controller at the wind farm will change the command on the output active power, which will lower the power injected by the wind farm to the DC system. This approach cannot be easily applied in engineering practices, because the communication devices must work rapidly, swiftly and safely, and the DC voltage will soar owing to communication delay.

\subsubsection{Increasing the Frequency}

When the DC voltage $U_{\mathrm{dc}}$ is below the threshold $U_{\mathrm{dcthr}}$ for the control action of frequency increase, the WFMMC operates in steady state. When the DC voltage $U_{\mathrm{dc}}$ surpasses the threshold, the WFMMC will start to increase the frequency: the output of the proportional controller of $\mathrm{DC}$ voltage will be added to the given amplitude $f_{\mathrm{WFN}}$ of the $\mathrm{AC}$ frequency at the sending end to elevate the reference frequency $f_{\mathrm{WFref}}$ of the wind farm. The frequency increment depends on the increment of the $\mathrm{AC}$ voltage [12]. The traditional frequency increase control strategy of the WFMMC is illustrated in Fig. 11.

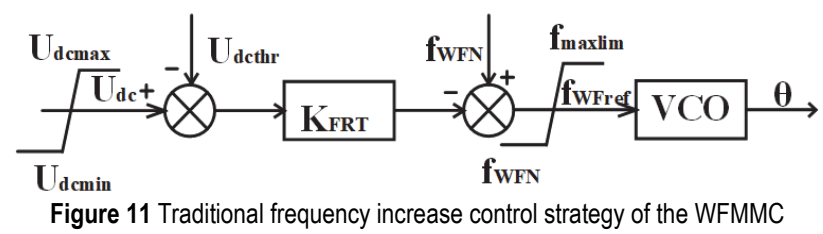

If the frequency increment is too large, the wind power generators might not maintain stable operation, and even trip. Hence, the variation of the AC frequency at the wind farm must be controlled within a certain range:

$K_{\mathrm{FRT}}=\frac{f_{\max \lim }-f_{\mathrm{WFN}}}{U_{\mathrm{dcmax}}-U_{\mathrm{dcthr}}}$

where, $U_{\mathrm{dcmax}}$ is the upper limit of the DC current of the $\mathrm{MMC} ; f_{\text {maxlim }}$ is the upper limit of the frequency increment.

\subsubsection{Reducing the Voltage}

The voltage reduction method changes the voltage command of the fixed AC voltage control at the converter station, according to the increment of DC voltage; then, the AC voltage of the offshore wind farm will be properly lowered to limit the power injected by the wind farm to the converter station. The voltage reduction at the converter station can be described as [6]:

$$
\begin{aligned}
& U_{\text {ac_ord }}= \\
& =\left\{\begin{array}{c}
U_{\text {ac_nom }} U_{\mathrm{dc}}<U_{\mathrm{dcthr}} \\
U_{\text {ac_nom }}-k_{\mathrm{v}}\left(U_{\mathrm{dc}}-U_{\mathrm{dcthr}}\right) U_{\mathrm{dcthr}} \leq U_{\mathrm{dc}} \leq U_{\mathrm{dcmax}} \\
U_{\text {acmin }} \\
U_{\mathrm{dc}}>U_{\mathrm{dcmax}}
\end{array}\right.
\end{aligned}
$$


where, $k_{\mathrm{v}}=\frac{U_{\mathrm{ac} \_ \text {nom }}-U_{\mathrm{acmin}}}{U_{\text {dcthrdcmax }}}$, which determines the reduction rate of the voltage; $U_{\text {ac nom }}$ is the reference $\mathrm{AC}$ voltage at the PCC of the wind farm; $U_{\mathrm{dc}}, U_{\mathrm{dcmax}}$ and $U_{\mathrm{dcthr}}$ have of the same meaning as in the frequency increase method; $\mathrm{U}_{\mathrm{acmin}}$ is the minimum $\mathrm{AC}$ voltage.

Both voltage reduction and frequency increase mitigate the excess power of flexible DC system at the cost of the stability of wind power generators. However, neither of them can fully solve the DC overvoltage phenomenon. $\mathrm{Li}$ et al. [13] studied the influence of voltage reduction and frequency increase over the wind power generators in wind farms with ordinary asynchronous generators / doubly fed generators, and drew the following laws:

(1) If the generators have a good LVRT ability, voltage reduction should be adopted to prevent ordinary asynchronous generators, which are oversensitive to frequency, from entering islanded mode. In this case, the frequency increase method would harm the rotor converter of doubly fed generators.

(2) If the generators have a poor LVRT ability, frequency increase should be adopted to stabilize the voltage at the wind farm.

Considering the limited regulation range of voltage and frequency at wind farms, frequency increase and voltage reduction are often coupled with other control strategies or unloading resistor. Zhu et al. [14] presented an assisted unloading control strategy coupled with voltage reduction method: an additional rotor current control was supplemented to the controller, such that the generators would not produce transient overcurrent in the case of too small voltage decrement.

\subsection{FRT}

Most of the MMC SMs in engineering applications adopt the half-bridge SM structure proposed by $\mathrm{R}$. Marquardt in 2002. Faced with any DC fault, the MMC, which is based on half-bridge SMs, the cut-in SMs like DC energy storage capacitor and the AC system will continuously inject the fault current to the DC side. When the MMC bridge arm current is found to surpass the limit, the SMs will be locked immediately, and the DC capacitor will be bypassed. Then, the MMC will exhibit the feature of uncontrollable rectification.

To solve the above problem, the traditional way is to apply an AC circuit breaker to isolate the AC system from the DC fault point, and then cut off the fault line using DC switches on the line. The long action time poses a challenge to the carrying ability of the converter and the restoration of the system. The converter station on the side of wind farm provides $\mathrm{AC}$ voltage and frequency supports to the wind farm. If it is locked, the wind farm will lose all the supports, and face power outage. Therefore, the converter station is expected to work uninterruptedly during DC fault to maintain the power transmission between the wind farm and the DC system, that is, have a certain ability of DC FRT. The current research on DC FRT mainly focuses on the application of DC circuit breakers and the improvement of MMC topology [15].

\subsubsection{Applying DC Circuit Breaker}

It is an effective and direct approach to isolate DC fault with DC circuit breaker. At present, there are three types of DC circuit breakers: mechanical, all-solid-state, and mixed. Song et al. [16] proposed an MMC embedded with circuit breakers, and proved it as a cost-effective solution to the elimination and isolation of DC fault: the DC fault current could be suppressed effectively through the coordination between the SM bypassing and DC circuit breaking. Based on DC voltage change rate, Zhang et al. [17] provided a DC fault protection method for the halfbridge flexible DC grid with mixed DC breakers. Song et al. [18] designed an MMC topology integrating the function of DC breakers: in the event of a DC fault, the converter control cooperates with the DC breaker control to remove the energy from the fault current to the energy absorption branch parallel to the DC buses.

\subsubsection{Improving MMC Topology}

Peng et al. [19] presented the topology of cell-bridge MMC composed of half-bridge SM and full-bridge SM (Fig. 11), which can eliminate DC fault without using DC circuit breakers. During the FRT process, the cell-hybrid $\mathrm{MMC}$ controls the mean voltage of capacitors. However, the voltages of capacitors on different bridges or valves are imbalanced and impossible to be controlled independently, and some SMs might face overvoltage. After analyzing the dynamic process of capacitor voltage through the FRT, Wang et al. adopted an assisted capacitor voltage control loop on the six arms to directly regulate capacitor voltage, thereby reducing the chance of overvoltage on SMs. Wang et al. [20] derived a clamp dual capacitor submodule (CDCSM) based on two-way switch. The CDCSM contains two capacitors, and outputs three levels. The fault current can be quickly blocked by adding the capacitors into the fault loop. Based on the traditional half-bridge MMC topology, Fan et al. [21] integrated the full-bridge SMs to the circuit to absorb the power to the inside of the MMC. Besides, assistive branches were added to the bridge arms and the DC side to quickly erase DC faults. Wang et al. [22] proposed an improved capacitive SM topology capable of automatically eliminating AC-side faults, and presented the design plan for the MMC bridge arm that mixes the new SMs with half-bridge SMs. In addition, the proposed SMs were compared in details with common $\mathrm{SMs}$ in terms of voltage resistance and economy.

\section{CONCLUSIONS}

Modular multi-level flexible DC transmission has become the first choice for long-distance transmission of large-scale wind farms. Based on the demonstration of DC transmission project of offshore wind power in Sheyang County, eastern China's Jiangsu Province, this paper designs the control strategies for the steady-state operations of the wind farm side and the system side, respectively, aiming to ensure that largescale generators can send out the power via the flexible DC system safely, reliably, and stably, and to maintain the voltage constant in the DC lines. Simulation results show that the flexible DC transmission system realized the reliable and efficient 
integration of wind power generated by the wind farm; the electric quantities on the AC or DC side remained stable, and the system operated well, despite the changes of generator output. Since the FRT ability is an important metric of system reliability, the authors analyzed the causes for $\mathrm{AC} / \mathrm{DC}$ faults of the wind power integration via flexible DC transmission system, and summarized some of the current solutions to these faults.

\section{Acknowledgements}

2021 Autonomous Region's major science and technology project "Key technologies for efficient integration and flexible control of high-power wind hydrogen storage systems".

\section{REFERENCES}

[1] 2020-2060 China's wind power installed capacity and $\mathrm{CO}_{2}$ forecast.

[2] Yahiaoui, A., Iffouzar, K., Ghedamsi, K., \& Himour, K. (2021). Dynamic performance analysis of VSC-HVDC based modular multilevel converter under fault. Journal Européen des Systèmes Automatisés, 54(1), 187-194. https://doi.org/10.18280/jesa.540121

[3] Liu,T. Q., Tao,Y., \& Li, B. H. (2017). Critical problems of wind farm integration via MMC-MTDC system. Power System Technology, 41(10), 3251-3260. https://doi.org/10.13335/j.1000-3673.pst.2017.1666

[4] Chen, X. (2014). Research on Isolated Island Operation Control Strategy of Offshore Wind Farm under Typical Fault. South China University of Technology.

[5] Li, Y. (2014). Study on MMC-HVDC Simulation Modeling for Wind Farm Access. South China University of Technology.

[6] Qi, D. L. (2019). Research on Modeling and Control Strategy of Wind Power Grid Connected System Based on $M M C$. Shenyang University of Technology.

[7] Li, J. H. (2019). Study on fault traversing strategy of offshore wind farm connected to flexible direct current grid. South China University of Technology.

[8] Cheah-Mane, M., Sainz, L., Liang, J., Jenkins, N., \& UgaldeLoo, C. E. (2017). Criterion for the electrical resonance stability of offshore wind power plants connected through HVDC links. IEEE Transactions on Power Systems, 32(6),4579-4589. https://doi.org/10.1109/TPWRS.2017.2663111

[9] Dang, R. (2019). Study on fault traversing strategy of wind farmgrid - connection based on MMC-HVDC. Nanjing University of Science and Technology.

[10] Liu, X., Song, Q., \& Liu, W. H. (2016). Acoordinated control strategy for faultride - through of offshore wind farm connected flexible HVDC transmission system. Electrical Measurement \& Instrumentation, 53(19), 1-6. https://doi.org/10.3969/j.issn.1001-1390.2016.19.001

[11] Zhang, Q. Z., Wang, B., Li, Y., \& Liu, C. (2020). Research on fault crossing coordination control of a wind farm via a flexible direct current transmission system. Power System Protection and Control, 48(10), 131-138. https://doi.org/10.19783/j.cnki.pspc.190769

[12] Li, Y. T. (2019). Study on fault traverse control strategy of doubly - fed wind farm through flexible direct gridconnection. Harbin Institute of Technology.

[13] Li, X., Song, Q., \& Liu, W. (2015). Impact of fault ride through methods on wind power generators in a VSCHVDC system. Automation on Electric Power Systems, 39(11), 31-36. https://doi.org/10.7500/AEPS20140515002

[14] Zhu, M., Li, W. X., Chao, P. P., Xu, D. G., \& Li, Y. T. (2018). Coordinated Control Strategy to Enhance LVRT Capability of MMC-HVDC Systems Connected Wind Farms. Automation of Electric Power Systems, 42(19), 77-82. https://doi.org/10.7500/AEPS20171120005

[15] Luo, Y. Y., Wang, Y. H., Song, R. H., Bi, J. T., Ye, W., \& Min, Y. X. (2021). An improved capacitive submodule with DC fault self-cleaning capability. High Voltage Technology.

[16] Song, Q., Zeng, R., Yu, Z., Liu, W., Huang, Y., Yang, W., \& $\mathrm{Li}$, X. (2018). A modular multilevel converter integrated with DC circuit breaker. IEEE Transactionson Power Delivery, 33(5), 2502-2512. https://doi.org/10.1109/TPWRD.2018.2815550

[17] Zhang, J. J. (2018). Research on DC Fault Protection Method of Flexible DC Power Grid with DC Circuit Breaker. Huazhong University of Science andTechnology.

[18] Song, Q., Yang, W. B., Li, X. Q., Li, J. G., Zeng, R., Huang, Y. L., She, Z. Q., \& Liu, W. H. (2017). An MMC Topology Integrated With DC Circuit Breaker. Proceedings of the CSEE, 37(20), 6004-6013. https://doi.org/10.13334/j.0258-8013.pcsee.171497

[19] Peng, M., Liu, H., Zhao, X., Xu, J., \& Yuan, J. (2020). Direct Capacitor Voltage Control Method of Hybrid MMC during DC Short Circuit Fault .2020 4th International Conference on HVDC (HVDC), 613-615. https://doi.org/10.1109/HVDC50696.2020.9292882

[20] Wang, C., Xu, T., Wang, Y., Tao, J. Y., Wei, X. G., \& Wang, X. Y. (2021). MMC clamp double capacitor submodule with the ability to block DC fault current. High Voltage Technology.

[21] Fan, Q., Zhao, X. B., \& Zhao, C. Y. (2021). Parallel hybrid MMC for DC grid fault clearance. Proceedings of the CSEE, 1-10. https://doi.org/10.13334/j.0258-8013.pcsee.201679

[22] Wang, Y., Fu, Y., Zeng, Q., \& Song, Y. (2019). Review on key techniques for fault protection of flexible DC grids. High Voltage Engineering, 45(8),2362-2374.

\section{Contact information}

Hua LI

School of Energy and Power Engineering,

Inner Mongolia University of Technology,

Hohhot 010051, China

E-mail: lihua0806@qq.com

\section{Keqilao MENG}

(Corresponding author)

School of Energy and Power Engineering, Inner Mongolia University of Technology,

Hohhot 010051, China

E-mail:2151708609@qq.com

\section{Xiaoyan LI}

School of Electric Power,

Inner Mongolia University of Technology,

Hohhot 010051, China

E-mail: 2326591855@qq.com

\section{Yufei PENG}

School of Electric Power,

Inner Mongolia University of Technology,

Hohhot 010051, China

E-mail: 2900154013@qq.com 